\title{
Likely detection of pulsed high-energy $\gamma$-rays from millisecond pulsar PSR J0218+4232
}

L. Kuiper, W. Hermsen

SRON-Utrecht, Sorbonnelaan 2, 3584 CA, Utrecht, The Netherlands

F. Verbunt

Astronomical Institute Utrecht, 3508 TA, Utrecht, The Netherlands

A. Lyne, I. Stairs

University of Manchester, Jodrell Bank, Macclesfield SK11 9DL, UK

D.J. Thompson

Code 661, LHEA, NASA GSFC, Greenbelt, MD 20771, USA

G. Cusumano

IFCAI CNR, Via U.La Malfa 153, I-90146, Palermo, Italy

\begin{abstract}
We report on the likely detection of pulsed high-energy $\gamma$ rays from the binary millisecond pulsar PSR J0218+4232 in 100-1000 $\mathrm{MeV}$ data from CGRO EGRET. Imaging analysis demonstrates that the highly significant $\gamma$-ray source 2EG J0220+4228 $(\sim 10 \sigma)$ is for energies $>100 \mathrm{MeV}$ positionally consistent with both PSR J0218+4232 and the BL Lac 3C66A . However, above $1 \mathrm{GeV} 3 \mathrm{C} 66 \mathrm{~A}$ is the evident counterpart, whereas between 100 and $300 \mathrm{MeV}$ PSR J0218+4232 is the most likely one. Timing analysis using one ephemeris valid for all EGRET observations yields in the $100-1000 \mathrm{MeV}$ range a double-pulse profile at a $\sim 3.5 \sigma$ significance level. The phase separation is similar to the component separation of $\sim 0.47$ observed at X-rays. A comparison of the $\gamma$-ray profile with the $610 \mathrm{MHz}$ radio profile in absolute phase shows that the two $\gamma$ - ray pulses coincide with two of the three emission features in the complex radio profile.
\end{abstract}

\section{Introduction}

PSR J0218+4232 was discovered by Navarro et al. (1995) as a 2.3 ms radiopulsar in a two day orbit around a low mass $\left(\sim 0.2 \mathrm{M}_{\odot}\right)$ white dwarf companion. A striking feature was that the radio-profile appeared complex and very broad.

Targeted observations at soft X-rays $(0.1-2.4 \mathrm{keV})$ with the ROSAT HRI instrument revealed also the pulsed nature in the soft X-ray window: a double 


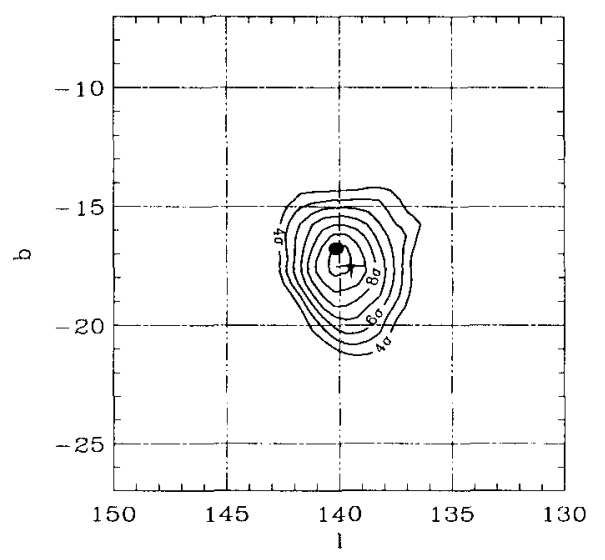

Figure 1. MLR image in galactic coordinates for energies in excess of $100 \mathrm{MeV}$ of the sky region containing $2 \mathrm{EG}$ J0220+4228, combining data from 5 separate observations. A detection significance of $z$ $10 \sigma$ is reached. The contours start at $4 \sigma$ in steps of $1 \sigma$ for 1 degree of freedom. PSR $\mathrm{J} 0218+4232$ is indicated by a star symbol and $3 \mathrm{C} 66 \mathrm{~A}$ by a bullet.

peaked lightcurve with a main emission feature phase separated by $\sim 0.47$ from a second less prominent pulse (Kuiper et al. 1998).

In a recent observation at harder X-rays (1.6-10 keV) with the BSAX MECS instruments the double peaked nature of the X-ray profile was confirmed (Mineo et al. 2000; Nicastro, these proceedings). Spectral analysis showed that the pulsed emission has a very hard spectrum with a power-law photon-index of $\sim-0.6$.

At high-energy $\gamma$-rays the positional coincidence of $2 \mathrm{EG}$ J0220+4228 (Thompson et al. 1995) with PSR J0218+4232 was noticed by Verbunt et al. (1996). These authors found also indications for pulsed emission at energies above $100 \mathrm{MeV}$. In this work all available EGRET observations of PSR J0218+4232 between April 1991 and November 1998 with off-axis angles $<30^{\circ}$ have been used to obtain maximum statistics. In the timing analysis we used one single very accurate ephemeris (rms error $85 \mu \mathrm{s}$ ) having a validity interval of about 5 years.

\section{Imaging analysis}

We have combined data from CGRO viewing periods 15, 211, 325, 427 and $728.7 / 9$ and binned the measured $\gamma$-ray arrival directions in a galactic $0.5 \times 0.5$ grid after applying "standard" EGRET event selections. The measured distribution is compared with an expected model distribution, composed of galactic and extra-galactic diffuse model components and established high-energy $\gamma$-ray sources within a 30.0 radius around PSR J0218+4232, by applying a Maximum Likelihood Ratio (MLR) test for the presence of a source at each grid position (for more details see Kuiper et al. 2000).

The MLR-map for energies $>100 \mathrm{MeV}$ is shown in Fig. 1 with superimposed the positions of 2 candidate counterparts, PSR J0218+4232 and 3C66A. The detection significance of the $\gamma$-ray source reaches a $\gtrsim 10 \sigma$ level. The number of counts $(>100 \mathrm{MeV})$ assigned to this excess is $\sim 230$. We also produced MLRmaps in the broad "standard" EGRET differential energy windows: 100-300 $\mathrm{MeV}, 300-1000 \mathrm{MeV}$ and $1-10 \mathrm{GeV}$. The resulting location confidence contours of the $\gamma$-ray source are shown in Fig. 2 for all 3 energy windows. 


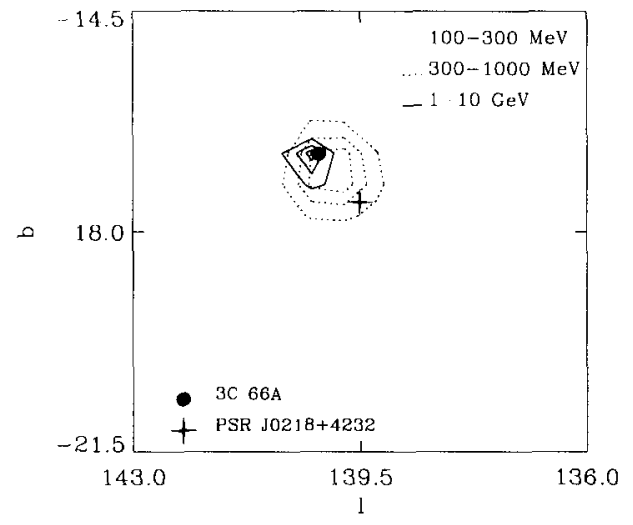

Figure 2. 1,2 and $3 \sigma$ location confidence contours of $\gamma$ ray source $2 \mathrm{EG} \mathrm{J} 0220+4228$ in three different broad energy intervals. Between 100-300 $\mathrm{MeV} 3 \mathrm{C} 66 \mathrm{~A}$ is located outside the $3 \sigma$ contour, whereas between $1-10 \mathrm{GeV}$ this is the case for PSR J0218+4232 .

This figure shows that $3 \mathrm{C} 66 \mathrm{~A}$ is the evident counterpart for the $1-10 \mathrm{GeV}$ window (consistent with the third EGRET catalogue result; Hartman et al. 1999), whereas PSR J0218+4232 is the most likely counterpart for the $100-300$ $\mathrm{MeV}$ window. Between 300 and $1000 \mathrm{MeV}$ both sources contribute to the excess.

\section{Timing analysis}

For the timing analysis we have selected events in a circular aperture around the PSR J0218+4232 position with an energy dependent extraction radius. This radius has been determined a priori from a signal-to-noise optimization study taking into account the energy dependent point source distribution and the best fit total sky background model as derived in the imaging analysis.

We folded the barycentric arrival times of $100-1000 \mathrm{MeV}$ events with the pulsar timing parameters from one single ephemeris taking into account the binary nature of the system. We obtained a $3.5 \sigma$ signal in a $Z_{4}^{2}$-test and the lightcurve showed one prominent pulse between phases 0.6 and 0.7 following a broader less prominent pulse between phases 0.1 and 0.4 (see Fig. 3b). In addition, a pulse phase resolved imaging analysis (see Kuiper et al. 2000) shows that the $100-300 \mathrm{MeV}$ spatial signal is concentrated in the 2 pulses.

A comparison with the X-ray BSAX MECS and ROSAT HRI lightcurves shows that the phase separation of the pulses in the $\gamma$-ray lightcurve is similar to the separation of $\sim 0.47$ found at X-rays.

Finally, we can compare the $100-1000 \mathrm{MeV}$ lightcurve in absolute phase with the $610 \mathrm{MHz}$ radio profile (Fig. 3a) and find that the $2 \gamma$-pulses coincide with 2 of the 3 radio-pulses within the CGRO absolute timing uncertainty of $\lesssim 100 \mu s$.

\section{Summary}

This study shows that we obtained good circumstantial evidence for the first detection of high-energy $\gamma$-rays from a millisecond pulsar, PSR J0218+4232 : 


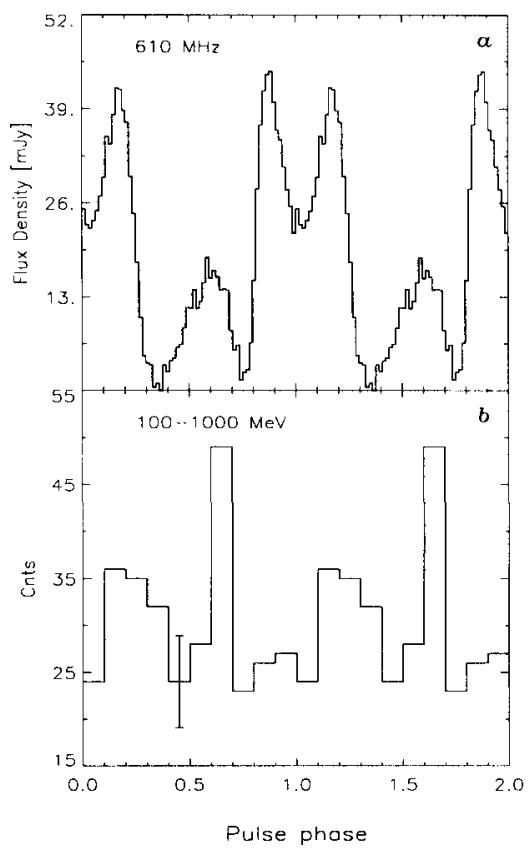

Figure 3. Comparison in absolute time of the radio 610 $\mathrm{MHz}$ profile (a; Stairs et al. 1999) and the $100-1000 \mathrm{MeV}$ EGRET lightcurve (b) of PSR J0218+4232. A typical error is indicated in the lower panel. Notice the (near) alignment of the 2 high-energy pulses with 2 of the 3 radio-pulses.

- A double-peaked lightcurve in the $100-1000 \mathrm{MeV}$ energy interval with a $\sim 3.5 \sigma$ modulation significance.

- The phase separation of the $2 \gamma$-pulses is similar to that at hard X-rays; a comparison in absolute time with the $610 \mathrm{MHz}$ radio-profile shows alignment of the $\gamma$ pulses with 2 of the 3 radio pulses.

- Between 100 and $300 \mathrm{MeV}$ the EGRET source position is consistent with PSR J0218+4232 with the signal concentrated in 2 pulses.

The full analysis and implications of our findings will be presented in detail in Kuiper et al. 2000.

\section{References}

Hartman R.C., Bertsch D.L., Bloom S.D., et al. 1999, ApJS 123, 79

Kuiper L., Hermsen W., Verbunt F., et al., 1998, A\&A 336, 545

Kuiper L., Hermsen W., Verbunt F., et al., 2000, A\&A, in preparation

Mineo T., Cusumano G., Kuiper L., et al., 2000, A\&A submitted

Navarro J., de Bruyn A.G., Frail D.A., et al., 1995, ApJ 455, L55

Stairs I.H., Thorsett S.E., Camilo F., 1999 , ApJS 123, 627

Thompson D.J., Bertsch D.L., Dingus B.L., et al., 1995, ApJS 101, 259

Verbunt F., Kuiper L., Belloni T., et al., 1996, A\&A 311, L9 